\title{
Learning Surgical Interventions by Navigating in Virtual Reality Case Spaces
}

Piet Kommers, Steffan Rödel, Jan-Maarten Luursema, Bob Geelkerken, and Eelco Kunst

\author{
University of Twente, Faculty of Behavioral Sciences \\ Medical Spectrum Hospital \\ Kunst en v Leerdam \\ Enschede, The Netherlands
}

\begin{abstract}
Virtual Reality is becoming a serious candidate for a learning environment for complex skills like vascular interventions. The diagnostics, dimensioning and insertion of the endograft stent has been modeled as a decision making process and now faces its implementation in a VR learning space.
\end{abstract}

\section{Introduction}

The DIME project (Distributed Interactive Medical Exploratorium for 3D Medical Images) aims at conceptualizing, implementing and researching the effectiveness of a VR-based pre-surgical planning and teaching environment. The DIME explorations aim at a pre-surgical planning and teaching applications, which will most likely result in better post-surgical results, lower health care costs and increased efficiency in the training of fellow-surgeons. The project is a collaboration of a 'computer science' group from the University of Amsterdam (UvA)1, a 'medically oriented computer science' group from the Leiden University Medical Center (LUMC) ${ }^{2}$, a 'cognition' group from the University of Twente (UT) and medical specialists from LUMC) and The Medical Spectrum Twente.

\section{Surgical Training in Virtual Reality}

The surplus of surgical training skills as well as being oriented in medical disciplines like anatomy, histology, physiology etc is evident. The optimization of its professional training elements needs to most complete repertoire of learning technology like the media spectrum and the full repertoire of new learning paradigms.

\footnotetext{
${ }^{1}$ Prof Dr. P. Sloot (UvA) and Dr. E. Zudilova

2 Prof Dr. JHC Reiber (RUL) and Dr. J. Schaap
} 
The aim of this paper is to show the compatibility between the most advanced visualization methods currently feasible for the average desktop work stations [2]. The overall conception is that the clinical training will gradually be extended with VR learning systems, in order to make the supervised real operations more effective and safe.

\subsection{Relevance of Virtual Reality}

1. Surgical techniques have become increasingly complex, thus making the learning curve to master these techniques steeper and longer.

2. More complex intervention techniques are rapidly developed and introduced in the daily practice.

3. The conventional surgical teaching method is a close daily working relation between the experienced teacher (trainer) and the unskilled pupil (trainee).

4. In traditional teaching the steep learning curve takes place during the interaction with real patients.

5. The modern patient does not accept any mutilation attributed neither to the disease nor to the intervention.

6. It is clear that a perfect preoperative visualization and planning, and rehearsals of these interventions are essential.

7. This means that while there is an increased demand for surgical training, experienced surgeons have increasingly less time and opportunity to cope with this demand. A dedicated medical VE is badly needed to lift this burden from their shoulders.

8. Of importance is also the possibility to allow trainees to explore critical situations and to let them experiment with an underlying model of the phenomena and processes in the human body, without the stress of having to deal with an actual patient.

9. Virtual surgical tools should be available for life-long medical education and assessment of the surgical consultant.

10. Based upon the disappointing experiences with "Intelligent Tutoring Systems" in the "80ies we do not want to undertake the paradigm of "training dummy mannequins" as it lacks the notions of "continuous learning" and the "surgeon as active problem solver".

\subsection{The Urgency for Laparoscopic Interventions}

Nowadays the laparoscopic cholecystectomy is the preferred technique in many hospitals. However, the majority of the surgeons performing laparoscopic cholecystectomies are autodidactic. They heard about the technique on congresses. They visited clinical demonstrations in centres of excellence. Thereafter they planned the first procedures in their own hospital. It is not surprising that the results are not as good as reported in the literature in the early periods. The steep learning curve was moved on patients. A sufficient training and formal assessment of the surgical team 
before introducing the new technique into the hospital is not available. Moreover, more complex intervention techniques are rapidly developed and introduced in the daily practice. An example of this is the endovascular exclusion of infernal aortic aneurysms with an endograft. Cuijper recently reported in his thesis that only after an endovascular experience of 30 electively treated triple A the complication ratio is sloping down to acceptable levels. In the Netherlands only a few hospitals have such an experience. Also the first ruptured aortic aneurysms are treated in the endovascular way with a very good outcome.

However, this emergency procedure demands a large endovascular experienced team available during day and night. Gaining enough experience with this procedure is not possible in most of the hospitals in the Netherlands. Unfortunately, it is not possible to transport patient with a ruptured triple A to centres of excellence because of hemodynamical instability. In other words, the patients do not survive delay of treatment due to transportation. The next generation, more complex endografts with the possibility of perirenal sealing is underway. The results of the first clinical experiments came from "down under". It is clear that a perfect preoperative visualisation and planning and a dummy operation of the whole procedure is essential.

\subsection{The Urgency for Training in Virtual Reality}

The conventional and still actual surgical teaching method, introduced more than a century ago, is a close daily working relation between the practised teacher (a consultant surgeon) and the unskilled pupil (the surgical resident). Working-weeks of 70 to 90 hours were accepted and after 6 years of gaining theoretical and especially practical skills under direct supervision of the consultant the resident becomes a surgeon. Nowadays our society does not accept such long periods of formal learning and the working week is shortened to a maximum of 48 hours. This results in a $40 \%$ decline of directly supervised practical experience of residents in their first years of surgical training. Moreover, the government asked the surgical society to offer the basic surgical training in only 5 years!

On the other side the same government makes laws as the BIG and the WGBO. The surgeons are obliged to be qualified (formal licensed) and to be skilled and properly trained to offer and execute an intervention to or on a patient. Due to the "Schengen convention" there is a right of free establishing of the citizens of the European community in the participating countries. The formal training of resident and consultants in the European countries showed large diversities. Objective and proper methods for assessment and comparison of the outcome of the surgical training in the European countries are not available. It is clear that the conventional surgical teaching methods do not fulfil the demands of patients, the society, the government and the surgical profession. New training methods have to be developed.

The development of virtual reality surgical tools for theoretical and practical training and assessment of the resident is urgent [4]. Moreover, virtual surgical tools should be available for life-long medical education and assessment of the surgical consultant maintaining a high level of expertise and skills in the profession. This 
project intends to contribute to the steep increasing need for practical training and objective assessment for the surgeon in training.

\subsection{Goals of the DIME Project}

1. To create a VR learning environment that allows surgical trainees to both practice their skills in the 'Operating Room' Virtual Environment (VE) and enhance their understanding of the procedure under study by using the 'Library' VE or the peer-to-peer chat function [5].

2. DIME aims at identifying the more 'objective' training elements that need to be conveyed before the constructionistic learning starts. This is the reason that the first stage arranges teams with the various expertises that play a role for the definition of the anchoring points in the training of the future surgeon [7].

3. Most of the VR projects have invested in the actual building of the models and have no didactic interface yet. The DIME project sees this need and aims at defining a generic instructional method that intermediates between a VR medical model and a novice who needs to understand and optimize its functioning.

4. To specify and evaluate VR elements for the pre-clinical training phase of novices in artery surgery.

\section{A-priory Expert System for Risk-Evaluation of Endovascular Stent Prosthesis Placement}

The VREST $^{3}$ group has undertaken the formalization of dimensioning the stent orthesis for the AAA patients. In order to obtain an uncomplicated passage and a lasting exclusion of an infrarenal abdominal aorta aneurysm (AAA) through endovascular placement of a stent-prosthesis, one has to take into account many unique anatomical properties of the aortic-iliac-femoral trajectory and many unique properties of the stent prosthesis.

Because of this large amount of relevant anatomical and stent-prosthestic variables it is not easy, even for the experienced clinician to make the correct assessment. A validated stent-prosthesis expert system can offer support to the clinician in choosing between endovascular and transabdominal exclusion of the AAA. Such a system can also offer support in choosing the optimal type of stent-prosthesis, and in planning the procedure.

\footnotetext{
3 The members of the VREST team (VREST: Virtual Reality Educational Surgical Tools) (Rödel SGJ, Kunst EE, Teijink JAW, Herwaarden JA van, Berg JC van den, Oude Groothuis P, Moll F, Palen J van der, Huisman A, Det RJ van, Geelkerken RH)
} 


\subsection{Goal}

The goal of this prior study was the validation of a custom developed stentprosthesis expert system. The anatomical AAA criteria were measured in 202 patients from two endovascular centers. Every AAA was divided in ten segments; suprarenal aorta, infrarenal aorta, aneurysm, aorta bifuration, right, left and common iliac artery and common left and right artery. For each segment, the following characteristics were recorded: length, thrombus, sclerosis, angulation and configuration. These 202 AAA's were then judged by five endovascular trained clinicians on anatomical fitness for placing the stent prosthesis [6].

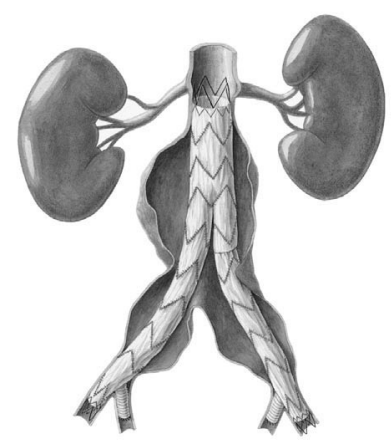

Fig. 1. The Stent Orthesis for the AAA Patients

Table 1. Correspondence Between the Clinicians and the Expert System

\begin{tabular}{lcccc} 
& Agreement & False positive & False negative & Discussion \\
\hline Surgeon 1 & 73.4 & 0.0 & 1.7 & 24.9 \\
Surgeon 2 & 75.5 & 0.7 & 2.5 & 21.3 \\
Surgeon 3 & 73.7 & 0.0 & 1.8 & 24.7 \\
Radiologist 1 & 70.6 & 0.5 & 4.2 & 24.8 \\
Radiologist 2 & 64.8 & 0.0 & 8.1 & 27.1 \\
\hline Total & $\mathbf{7 1 . 6}$ & $\mathbf{0 . 2}$ & $\mathbf{3 . 7}$ & $\mathbf{2 4 . 6}$ \\
\hline
\end{tabular}

Agreement: $\quad$ Same advice clinician and expert system.

False positive: $\quad$ Advice clinician $=$ no intervention, advice expert system $=$ intervention.

False negative: $\quad$ Advice clinician $=$ intervention, advice expert system $=$ no intervention.

Discussion: $\quad$ Advice clinician $=$ high risk intervention, expert system $=$ no intervention

The chances for successful sealing were independently expressed in 'complication rating' for each AAA by five clinicians and the expert program. The complication rating was divided in $0 \%$ to $59 \%$ (low to intermediate risk), $60 \%$ to $94 \%$ (intermediate to high risk), $95 \%$ to $98 \%$ (very high risk), $99 \%$ (practically impossible) and $100 \%$ (impossible) to obtain sealing. It was allowed to choose between all configurations of three commercially available stent-prostheses. A total of 3030 AAA assessments were 
given by the five clinicians. These were compared to the assessments of the expert system.

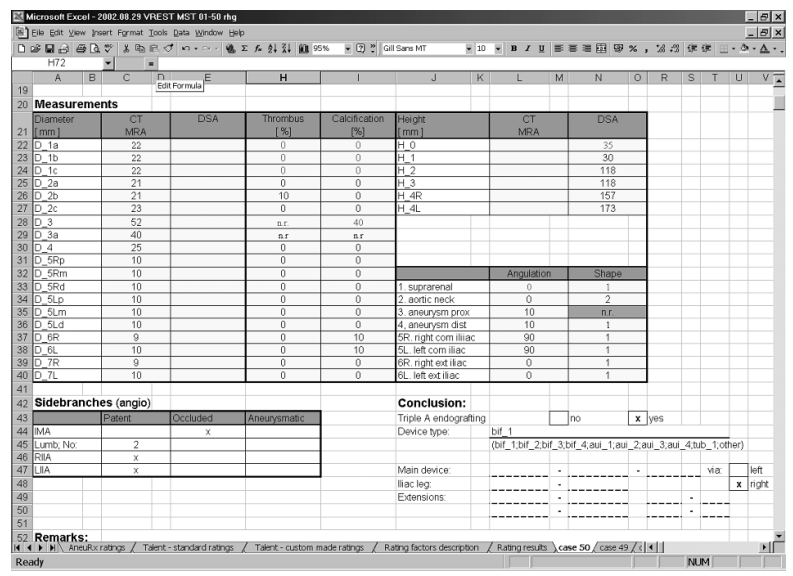

Fig. 2. Abdominal aorta aneurysm anatomical data input screen

\subsection{Results and Preliminary Conclusion}

There appeared to be a good correspondence between the assessments of the clinicians and the expert system as finally visualized as in Figure 2. Specifically the chances for an incorrect positive advice from the expert system are minimal (1:500 assessments). In case of a high complication rating the expert system tends to advise negative on stent placement in more cases then the experienced clinician does.

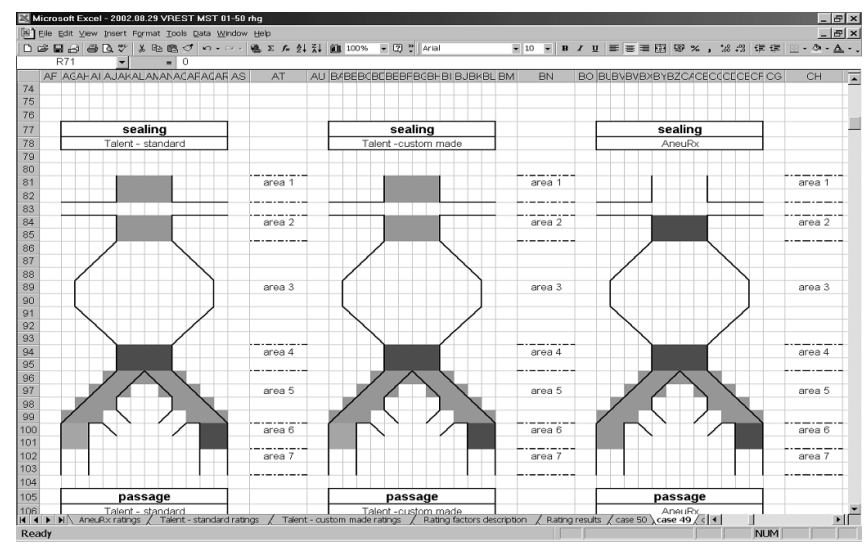

Fig. 3. Discrete Modeling Rational for the go-no go and the Dimensioning of the Stent Artifact

The basic underlying rational for the go-no go and the dimensioning of the stent artifact has been certified in this way. The envisaged DIME project plans the support 
of the $3 \mathrm{D}$ aspects of the particular patient in concern. Both the experienced and the novice vascular surgeon will increase effectiveness and task efficiency as the MRA images are transformed into three-dimensional models that can be inspected for critical morphologies and to anticipate better to the actual medical intervention.

\subsection{Initial Architecture of the Proposed VR Learning Context}

The surgical skills and its continuous sophistication; how should it benefit ideally from the virtual Operating Room? In this practice "space" (at the right side of Figure 2 ) the cycle goes around diagnosis, prepare and execute the intervention. But at unforeseen moments we expect that students consult a library of domain expertise. Based on the log data generated by this VE,

- the coach and trainee can evaluate his/her progress and set out an appropriate personal learning path

- $\quad$ the coach can extract general trends in learning of his/her trainees, as well as differences in learning styles of individual trainees.

Though the overall of this architecture looks adequate and robust, we decided not to adopt it basically, because of the suggested antagonism between the two [2]. More adequate and fair seemed the model where the learning between the various approaches due to variations in patients and the subsequent surgeon experts and the various trainees was the central core of the learning.

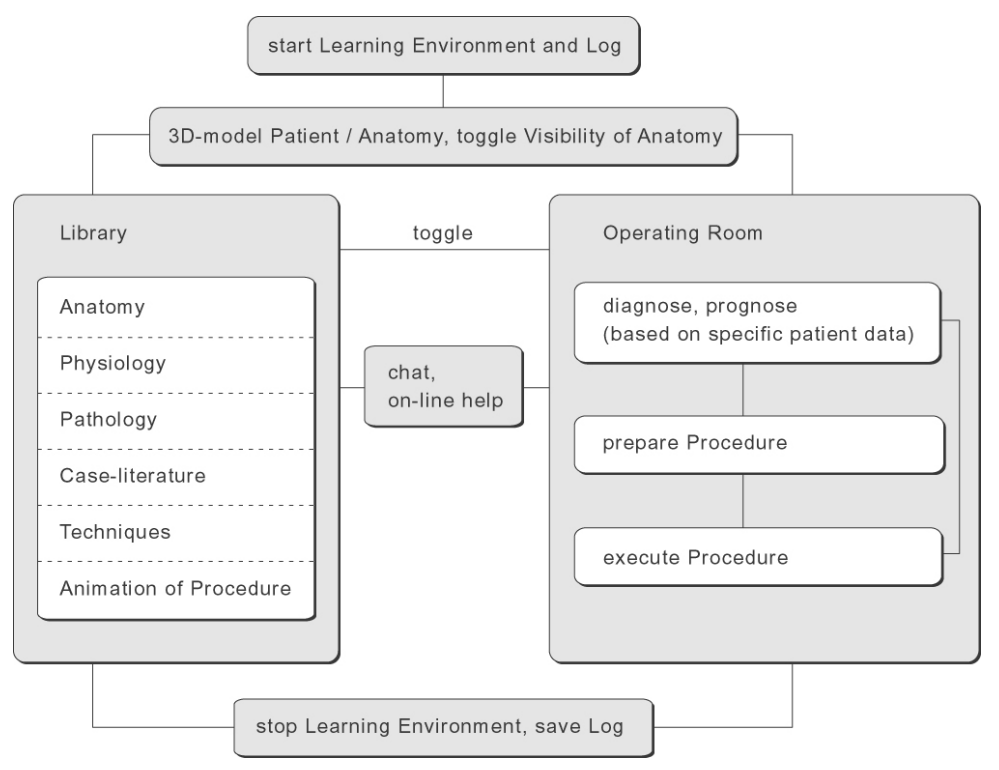

Fig. 4. The interplay between the students' theory versus skill-driven learning

The alternative to the theory/practice model above was left behind and the key idea for the VR training space was called "Interconnected Expertise". It aims at bring the 
novice in a quasi continuum of surgical interventions. Learning in this space allows a fluent transition between patients, surgeons and various stages in the intervention. The more natural one is by tracing the treatment by one surgeon in one patient through the natural chronology of the operation. But there are good reasons to switch between patients as it shows typical morphologies that clarify the reason why the initial treatment was needed there [1].

\section{Imagining VR as Interconnected Expertise}

VR as representation of the targeted object world may be propagated sufficiently. More intriguing is the question how learners may benefit from the prior experts and successful peer-learners. This question was recently addressed and came to the idea that three main dimensions need to be articulated before a meaningful navigation by the learner may take place.

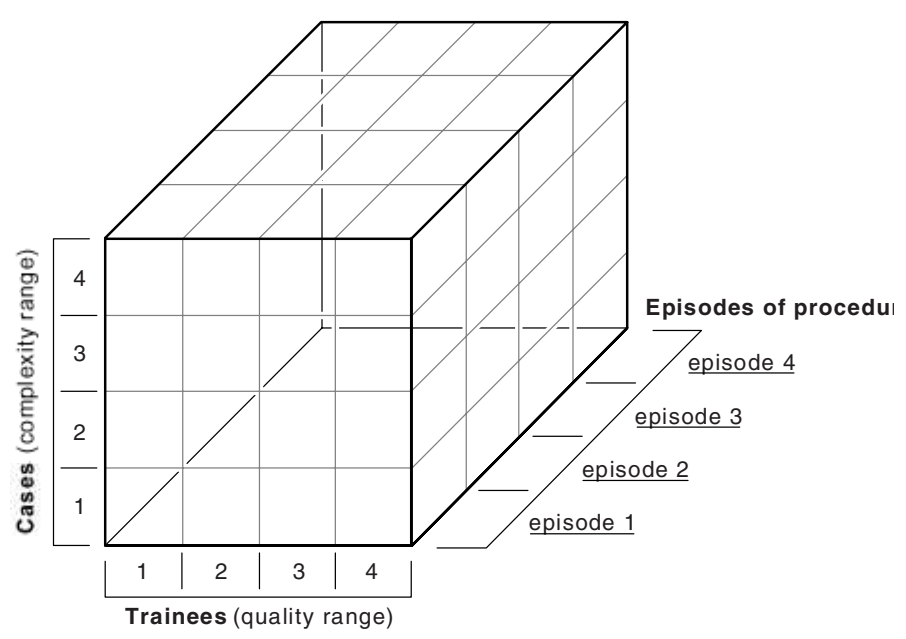

Fig. 5. Three main dimensions for allowing the learner to navigate in prior vascular interventions

1. The first dimension (called Cases) is the set of indexed patients who vary from obeying the prototypical standard medical problem as listed in the study books; in this case the abdominal aorta aneurism is clearly there, but with a minimum of complicated side effects and the patients is in a good overall condition. At the other end of the continuum there is the patient who suffers from a severe AAA phenomena, but at the same time a large number of constraining factors can be discerned like stenosis, aneurisms before and after the bifurcation and a complex artery morphology 
2. The second dimension (called Trainees) varies between the highlyexperienced vascular surgeons who performed in the Endograft stent VR model quite punctual and highly correct AAA interventions, to most of the embedded cases. At the other end of this dimension one can find recorded AAA interventions by freshmen demonstrating the many thinkable flaws. At each of the suboptimal interventions the ultimate surgeon marked the reason of labeling the flaw as being suboptimal and activates links to the real good solutions that should have been made.

3. The third dimension (called Episode 1..n) captures the subsequent stages in the AAA intervention. Two criteria for ordering them can be taken: The chronology of sub actions from early to later, versus the ordering from easy to complex. Analytical and experimental validation still needs to be performed in order to make a sensible choice here. In any case following the strict order of this dimension allows the learner to follow the prior interventions in it chronological order.

\section{Conclusion}

Though virtual reality is one of the prime candidates in vitalizing learning by its realism and direct appeal to the students' natural affordance to act upon urgencies rather than to "know" what experts are saying; VR in itself is not enough to make the learning more effective. By logic: Realism in VR does not suffice to exceed the real situation itself. We know from for instance link trainers for airplane pilots that the simulation can be more effective, once it elicits the novice to go into critically complex situations; exactly those situations that we never hope to meet in reality. Its added value is not only that the learner's reflexes are trained to survive in the panic of preciously decisive seconds. Its value is also that after all the fundamental understanding of complex mechanisms can best be understood if the learner is allowed to walk on the edge of what is a success versus a failure.

\section{References}

1. Beck K. and Cunningham W. (1989), A laboratory for teaching object-oriented thinking. In Proc. OOPSLA'89, ACM Sigplan Notices 17(4), pp. 1-6.

2. Kommers, P.A.M \& Zhiming, Z.; (1998) Conceptual Support with Virtual Reality in Webbased Learning. (Co-author Zhao Zhiming). In: International Journal of Continuing Engineering Education and Life-Long Learning. ISSN 0957-4344. Volume 8, No 1/2. pp 184-204.

3. Kommers, P.A.M.; (2003). Experiential Learning through Constructivist Learning Tools. In: International Journal of Computers and Applications, Vol. 25, No 1, 2003. (pp 1-12). ACTA Press. ISSN: 1206-212X (202).

4. Lanier, Jaron; (1992). Virtual Reality: The Promise of the Future. In: Interactive Learning International, v8 n4 p275-79 Oct-Dec 1992 ISSN: 0748-5743. 
5. McLellan, Hilary (1995) 6p.;Magical Stories: Blending Virtual Reality and Artificial Intelligence In: Imagery and Visual Literacy: Selected Readings from the Annual Conference of the International Visual Literacy Association (26th, Tempe, Arizona, October 12- 16, 1994).

6. Moshell, J.M., and Hughes, C.E. (1994, January). Shared Virtual Worlds for Education. Virtual Reality World, 2 (1), 63-74.

7. Psotka, Joseph; Immersive Training Systems: Virtual Reality and Education and Training. In: Instructional Science, v23 n5-6 p405-31 Nov 1995. ISSN: 0020-4277. 